\title{
Dynamics and Control of a Class of Underactuated Mechanical Systems
}

\author{
Mahmut Reyhanoglu, Member, IEEE, Arjan van der Schaft, Senior Member, IEEE, \\ N. Harris McClamroch, Fellow, IEEE, and Ilya Kolmanovsky, Member, IEEE
}

\begin{abstract}
This paper presents a theoretical framework for the dynamics and control of underactuated mechanical systems, defined as systems with fewer inputs than degrees of freedom. Control system formulation of underactuated mechanical systems is addressed and a class of underactuated systems characterized by nonintegrable dynamics relations is identified. Controllability and stabilizability results are derived for this class of underactuated systems. Examples are included to illustrate the results; these examples are of underactuated mechanical systems that are not linearly controllable or smoothly stabilizable.
\end{abstract}

Index Terms - Controllability, mechanical systems, nonlinear control, stabilizability, underactuated.

\section{INTRODUCTION}

$\mathbf{I}$ $\mathrm{N}$ THE past few years, there has been a surge of interest in the control of mechanical systems that satisfy certain nonintegrable relations. These studies were primarily limited to nonholonomic systems satisfying nonintegrable kinematics relations (see, e.g., [4], [15], and references therein). In this paper the ideas in [4] are extended to mechanical systems that satisfy nonintegrable dynamics or acceleration relations.

Systems with nonintegrable acceleration relations can arise by imposition of certain design conditions on the allowable motions of redundant manipulators. Such systems can also arise as models of underactuated mechanical systems, defined as systems which have fewer inputs than degrees of freedom. While many interesting techniques and results have been presented for underactuated systems, the control of these systems still remains an open problem. Important issues are: how can nonlinear control models be formulated for such systems; what are their controllability and stabilizability properties; and how can open-loop and closed-loop control problems be solved. The first two issues are thoroughly addressed in this paper, while the third issue is briefly discussed and references to published literature are provided. This paper is an extended version of the conference papers [25] and [26].

Manuscript received July 11, 1996; revised June 10, 1997 and May 6, 1998. Recommended by Associate Editor, O. Egeland. This work was supported in part by the Dutch Institute of Systems and Control and the NSF under Grant ECS-9625173.

M. Reyhanoglu is with the Department of Physical Sciences, Embry-Riddle Aeronautical University, Daytona Beach, FL 32114 USA.

A. van der Schaft is with the Department of Applied Mathematics, University of Twente, 7500 AE Enschede, The Netherlands.

N. H. McClamroch is with the Department of Aerospace Engineering, The University of Michigan, Ann Arbor, MI 48109 USA.

I. Kolmanovsky is with the Ford Research Laboratory, Dearborn, MI 48121 USA.

Publisher Item Identifier S 0018-9286(99)07130-5.

\section{Models of Underactuated Mechanical Systems}

Consider first a dynamic system defined on a configuration manifold Q. Let $(q, \dot{q})=\left(q^{1}, \cdots, q^{n}, \dot{q}^{1}, \cdots, \dot{q}^{n}\right)$ denote local coordinates on the tangent bundle $\mathbf{M}=\mathbf{T Q}$. We refer to $q, \dot{q}$, and $\ddot{q}$ as the vectors of generalized coordinates, generalized velocities, and generalized accelerations, respectively. Let the system be under the action of $m<n, m \geq 1$, independent control forces and/or torques, i.e., there are fewer control inputs than degrees of freedom. Also let $u \in \mathbf{R}^{m}$ denote the vector of control variables. We partition the set of generalized coordinates $q=\left(q^{1}, \cdots, q^{n}\right)$ as $q=\left(q_{1}, q_{2}\right), q_{1} \in \mathbf{R}^{m}, q_{2} \in$ $\mathbf{R}^{n-m}$. Without loss of generality, we assume that the actuated degrees of freedom are represented by the elements of $q_{1}$ and the unactuated degrees of freedom are represented by the elements of $q_{2}$. Lagrange's equations can then be written as

$$
\begin{aligned}
& M_{11}(q) \ddot{q}_{1}+M_{12}(q) \ddot{q}_{2}+F_{1}(q, \dot{q})=B(q) u \\
& M_{21}(q) \ddot{q}_{1}+M_{22}(q) \ddot{q}_{2}+F_{2}(q, \dot{q})=0
\end{aligned}
$$

where $B(q) \in \mathbf{R}^{m \times m}$ is invertible for all $q \in \mathbf{Q}, F_{1}(q, \dot{q}) \in$ $\mathbf{R}^{m}, F_{2}(q, \dot{q}) \in \mathbf{R}^{n-m}$, and $M_{i j}(q), i, j=1,2$, represent components of an $n \times n$ inertia matrix which is symmetric and positive definite for all $q \in \mathbf{Q}$. Throughout this paper all functions are assumed to be smooth $\left(C^{\infty}\right)$ functions defined on $\mathbf{M}$.

Following Spong [33], we may solve for $\ddot{q}_{2}$ as

$$
\ddot{q}_{2}=-M_{22}^{-1}(q)\left[M_{21}(q) \ddot{q}_{1}+F_{2}(q, \dot{q})\right]
$$

and substitute into (1) to obtain

$$
\bar{M}(q) \ddot{q}_{1}+\bar{F}(q, \dot{q})=B(q) u
$$

where

$$
\begin{aligned}
\bar{M}(q) & =M_{11}(q)-M_{12}(q) M_{22}^{-1}(q) M_{21}(q) \\
\bar{F}(q, \dot{q}) & =F_{1}(q, \dot{q})-M_{12}(q) M_{22}^{-1}(q) F_{2}(q, \dot{q}) .
\end{aligned}
$$

Consequently, using the partial feedback linearizing controller

$$
u=B^{-1}(q)[\bar{M}(q) v+\bar{F}(q, \dot{q})]
$$

(1) and (2) can be rewritten as

$$
\begin{aligned}
& \ddot{q}_{1}=v \\
& \ddot{q}_{2}=J(q) \ddot{q}_{1}+R(q, \dot{q})
\end{aligned}
$$

where

$$
\begin{aligned}
J(q) & =-M_{22}^{-1}(q) M_{21}(q) \\
R(q, \dot{q}) & =-M_{22}^{-1}(q) F_{2}(q, \dot{q}) .
\end{aligned}
$$


Equations (3) and (4) have a special triangular or cascade form that appropriately captures the important attributes of underactuated mechanical systems. Equation (3) defines the linearized dynamics of the $m$ completely actuated degrees of freedom. Equation (4) defines the dynamics of the $n-m$ unactuated degrees of freedom; these are expressed in terms of equalities involving the generalized accelerations. If these latter relations do not admit any nontrivial integral, i.e., any smooth function $h(q, \dot{q}, t)$ such that $d h / d t=0$ along the solutions, then these relations may be interpreted as $n-m$ completely nonintegrable acceleration constraints (or secondorder nonholonomic constraints [25]). As will be seen in the subsequent development, controllability and stabilizability properties of underactuated mechanical systems are closely related to this property. Hence, it is crucial to identify underactuated mechanical systems where the acceleration relations defined by (4) are completely nonintegrable.

Define the $n-m$-covector fields

$$
\begin{gathered}
\omega^{i}=\sum_{j=1}^{m} J_{i j}(q) d \dot{q}_{1, j}-d \dot{q}_{2, i}+R_{i}(q, \dot{q}) d t \\
i=1, \cdots, n-m
\end{gathered}
$$

on $\mathbf{M} \times \mathbf{R}$ so that the $n-m$ relations given by (4) can be rewritten as $\omega^{i}=0, i=1, \cdots, n-m$. Augment the covector fields (5) with

$$
\begin{aligned}
\tilde{\omega}^{j} & =d q_{1, j}-\dot{q}_{1, j} d t, \quad j=1, \cdots, m \\
\tilde{\omega}^{m+k} & =d q_{2, k}-\dot{q}_{2, k} d t, \quad k=1, \cdots, n-m
\end{aligned}
$$

and let $\Omega \subset \mathbf{T}^{*}(\mathbf{M} \times \mathbf{R})$ denote the codistribution

$$
\Omega=\operatorname{span}\left\{\omega^{i}, \tilde{\omega}^{j}, i=1, \cdots, n-m, j=1, \cdots, n\right\} .
$$

The annihilator of $\Omega$, denoted $\Omega^{\perp}$, is spanned by $m+1$ linearly independent smooth vector fields

$$
\begin{aligned}
\tau_{0}= & \sum_{j=1}^{m} \dot{q}_{1, j} \frac{\partial}{\partial_{q_{1, j}}}+\sum_{k=1}^{n-m}\left(\dot{q}_{2, k} \frac{\partial}{\partial_{q_{2, k}}}+R_{k}(q, \dot{q}) \frac{\partial}{\partial \dot{q}_{2, k}}\right) \\
& +\frac{\partial}{\partial t} \\
\tau_{j}= & \frac{\partial}{\partial \dot{q}_{1, j}}+\sum_{i=1}^{n-m} J_{i j}(q) \frac{\partial}{\partial \dot{q}_{2, i}}, \quad j=1, \cdots, m .
\end{aligned}
$$

We present the following definition.

Definition 1: Consider the distribution $\Omega^{\perp}$ and let $\tilde{\mathcal{C}}$ denote its accessibility algebra, i.e., the smallest subalgebra of $\mathbf{V}^{\infty}(\mathbf{M} \times \mathbf{R})$ that contains $\tau_{0}, \tau_{1}, \cdots, \tau_{m}$. Let $\tilde{C}$ denote the accessibility distribution generated by the accessibility algebra $\tilde{\mathcal{C}}$. Then the acceleration relations defined by (4) are said to be completely nonintegrable if

$$
\operatorname{dim} \tilde{C}(x, t)=2 n+1, \forall(x, t) \in \mathbf{M} \times \mathbf{R} .
$$

Note that the above definition gives a coordinate-free characterization of nonintegrability for any set of acceleration relations of the form (4). Clearly, Frobenius' theorem (see, e.g., [22]) applies here. Note also that this definition is analogous to the definition given in [4] for the nonintegrability of a set of kinematics or velocity relations.
Other definitions of nonintegrability have been given in [23] and [37]. The definition given in [23] applies to underactuated manipulators and involves a coordinate-dependent requirement, namely noncyclicity of the unactuated joint variables. The definition given in [37] applies to underactuated vehicle models where the acceleration relations are expressed in terms of quasicoordinates (see, e.g., [11]) rather than to models expressed in terms of generalized coordinates. Our subsequent examples illustrate cases for which the definitions given in [23] and [37] are not applicable.

In this paper it is assumed throughout that the acceleration relations described by (4) are completely nonintegrable. Note that both completely nonintegrable velocity relations and completely nonintegrable acceleration relations have in common the fact that they do not reduce the dimension of the configuration space. However, in contrast to the completely nonintegrable velocity relations, completely nonintegrable acceleration relations do not reduce the dimension of the state space as well. Note also that any mechanical system with holonomic or classical nonholonomic constraints can be expressed in the form of an underactuated mechanical system with integrable acceleration relations.

Examples of underactuated systems with completely nonintegrable acceleration relations include underactuated robot manipulators [1], [12], [18], [23], [34], underactuated marine vehicles [10], [27], [37], the planar vertical takeoff and landing aircraft [13], [19], the rotational translational actuator system [6], [14], [16], and the acrobot system [32] and examples in [20] and [30].

A particularly important class of solutions is the equilibrium solutions of (3) and (4) with $v(t)=0, \forall t \geq 0$. A solution is an equilibrium solution if it is a constant solution; note that if $(q, \dot{q})=\left(q^{e}, 0\right)$ is an equilibrium solution we refer to $q^{e}$ as an equilibrium configuration. Clearly, the set of equilibrium configurations of system (3) and (4) is given by

$$
\{q \in Q \mid R(q, 0)=0\} \text {. }
$$

Equations (3) and (4) can be expressed in the usual nonlinear control system form by defining the following state variables:

$$
x_{1}=q_{1}, \quad x_{2}=q_{2}, \quad x_{3}=\dot{q}_{1}, \quad x_{4}=\dot{q}_{2} .
$$

Then the state equations are given by

$$
\begin{aligned}
& \dot{x}_{1}=x_{3} \\
& \dot{x}_{2}=x_{4} \\
& \dot{x}_{3}=v \\
& \dot{x}_{4}=J\left(x_{1}, x_{2}\right) v+R\left(x_{1}, x_{2}, x_{3}, x_{4}\right) .
\end{aligned}
$$

Equations (11)-(14) define a drift vector field $f(x)=$ $\left(x_{3}, x_{4}, 0, R\left(x_{1}, x_{2}, x_{3}, x_{4}\right)\right)$ and control vector fields $g_{i}(x)=\left(0,0, e_{i}, J_{i}\left(x_{1}, x_{2}\right)\right)$, where $e_{i}$ denotes the $i$ th standard basis vector in $\mathbf{R}^{m}$ and $J_{i}\left(x_{1}, x_{2}\right)$ denotes the $i$ th column of the matrix function $J\left(x_{1}, x_{2}\right), i=1, \cdots, m$, according to the standard control system form

$$
\dot{x}=f(x)+\sum_{i=1}^{m} g_{i}(x) v_{i} .
$$


Note that an equilibrium solution $x^{e}$, corresponding to $v=$ 0 , of (15) has the form $x_{1}^{e} \in \mathbf{R}^{m}, x_{2}^{e} \in \mathbf{R}^{n-m}$, where $R\left(x_{1}^{e}, x_{2}^{e}, 0,0\right)=0$, and $x_{3}^{e}=x_{4}^{e}=0$, i.e., an equilibrium solution corresponds to a motion of the system for which all the configuration variables remain constant. The controllability and stabilizability properties of system (3) and (4) near an equilibrium configuration $q^{e}$ can be obtained by studying local properties of system (11)-(14) near the corresponding equilibrium solution $\left(x_{1}^{e}, x_{2}^{e}, 0,0\right)$.

\section{Controllability AND Stabilizability Results}

This section develops controllability and stabilizability results for underactuated systems with completely nonintegrable acceleration relations. The reader is referred to [3], [22], and [35] for the controllability concepts developed in the nonlinear control literature.

We first demonstrate that an underactuated mechanical system, defined by (3) and (4), does satisfy certain nonlinear controllability properties. In particular, we show that the system is strongly accessible. This nonlinear controllability property is equivalent to Definition 1 and it guarantees that a necessary condition for small time local controllability (STLC) of the system at the equilibrium is satisfied.

Theorem 1: Let $n-m \geq 1$. The underactuated mechanical system, defined by (3) and (4), is strongly accessible.

Proof: Since we have assumed that relations (4) are completely nonintegrable the distribution $\Omega^{\perp}$ spanned by $\tau_{0}, \tau_{1}, \cdots, \tau_{m}$ satisfies the accessibility Lie algebra rank condition at any $(q, \dot{q}, t) \in \mathbf{M} \times \mathbf{R}$, i.e.,

$$
\operatorname{dim} \tilde{C}(x, t)=2 n+1, \forall(x, t) \in \mathbf{M} \times \mathbf{R} .
$$

Let $\pi_{M}: \mathbf{M} \times \mathbf{R} \rightarrow \mathbf{M}$ denote the projection onto $\mathbf{M}$. Then, clearly $\pi_{M^{*}} \tau_{0}=f$ and $\pi_{M^{*}} \tau_{i}=g_{i}, i=1, \cdots, m$. Let $\mathcal{C}_{0}$ denote the strong accessibility algebra associated with $f, g_{i}, i=1, \cdots, m$, i.e., the smallest subalgebra which contains $g_{1}, \cdots, g_{m}$ and satisfies $[f, X] \in \mathcal{C}_{0}, \forall X \in \mathcal{C}_{0}$, and let $C_{0}$ denote the strong accessibility distribution generated by the strong accessibility algebra $\mathcal{C}_{0}$. Since we have

$$
\operatorname{dim} \tilde{C}(x, t)=\operatorname{dim} C_{0}(x)+1
$$

it follows that

$$
\operatorname{dim} C_{0}(x)=2 n, \quad \forall x \in \mathbf{M} .
$$

Hence, system (11)-(14) is strongly accessible. Consequently, the underactuated mechanical system, defined by (3) and (4), is strongly accessible.

The following result illustrates the fact that for certain underactuated mechanical systems a given equilibrium configuration cannot be asymptotically stabilized using timeinvariant continuous (static or dynamic) state feedback. This property has been previously recognized for underactuated manipulators [23] and for underactuated vehicles [37].

Theorem 2: Assume that $R_{i}(q, 0)=0, \forall q \in \mathbf{Q}$, for some $i \in\{1, \cdots, n-m\}$. Let $n-m \geq 1$ and let $\left(q^{e}, 0\right)$ denote an equilibrium solution. Then the underactuated mechanical system, defined by (3) and (4), is not asymptotically stabilizable to $\left(q^{e}, 0\right)$ using time-invariant continuous (static or dynamic) state feedback law.
Proof: A necessary condition for the existence of a timeinvariant continuous asymptotically stabilizing state feedback law for system (11)-(14) is that the image of the mapping

$$
\begin{aligned}
& \left(x_{1}, x_{2}, x_{3}, x_{4}, v\right) \\
& \quad \mapsto\left(x_{3}, x_{4}, v, J\left(x_{1}, x_{2}\right) v+R\left(x_{1}, x_{2}, x_{3}, x_{4}\right)\right)
\end{aligned}
$$

contains some neighborhood of zero (see Brockett [5]). No points of the form

$$
(000, \epsilon), \epsilon \neq 0
$$

are in its image; it follows that the necessary condition is not satisfied. Hence system (11)-(14) cannot be asymptotically stabilized to $\left(x_{1}^{e}, x_{2}^{e}, 0,0\right)$ by a time-invariant continuous (static or dynamic) state feedback law. Consequently, the underactuated mechanical system, defined by (3) and (4), is not asymptotically stabilizable to $\left(q^{e}, 0\right)$ using a time-invariant continuous (static or dynamic) state feedback.

There are numerous examples of underactuated mechanical systems for which the assumption of Theorem 2 is not satisfied; in such cases an equilibrium solution may be smoothly (even linearly) stabilizable.

It is well known that strong accessibility is far from being sufficient for the existence of a feedback control which asymptotically stabilizes the underactuated system at an equilibrium solution. In certain cases it is possible to prove a stronger controllability property such as STLC, which guarantees the existence of a piecewise analytic feedback law for asymptotic stabilization in the real analytic case [36] (see the remark below). Since an underactuated mechanical system satisfies $1 \leq m<n$, the dimension of the state is at least four. Hence, in the real analytic case, the STLC property also guarantees the existence of asymptotically stabilizing continuous timeperiodic feedback laws [8, Th. 1.4]. We consider the definition of "asymptotic feedback stabilization" as given in [36], which involves a specification of "exit rules" for certain lowerdimensional submanifolds. Our assertion above is based on the result in [36] and is not in contradiction with the result in [31], which states that when the system's solutions are defined in the sense of Fillippov, the existence of stabilizing piecewise analytic feedbacks implies the existence of continuous time-invariant feedbacks. Indeed, the definition of asymptotic stability in [31] differs from that in [36]. We refer the reader to [7] for a detailed discussion on the relationship between controllability and feedback stabilization.

We now briefly summarize a result of Bianchini and Stefani [3], which we utilize to prove the subsequent controllability results. Let $\operatorname{Br}(X)$ denote the smallest Lie algebra of vector fields containing $f, g_{1}, \cdots, g_{m}$ and let $B$ denote any bracket in $B r(X)$. Let $\delta^{0}(B), \delta^{1}(B), \cdots, \delta^{m}(B)$ denote the number of times $f, g_{1}, \cdots, g_{m}$, respectively, occur in the bracket $B$. For an admissible weight vector $\mathbf{l}=\left(l_{0}, l_{1}, \cdots, l_{m}\right), l_{i} \geq$ $l_{0} \geq 0, \forall i$, the l-degree of $B$ is equal to the value of $\sum_{i=0}^{m} l_{i} \delta^{i}(B)$. The Bianchini and Stefani condition for STLC for a strongly accessible system is essentially that the so-called bad brackets, the brackets with $\delta^{0}(B)$ odd and $\delta^{i}(B)$ even for each $i$, must be l-neutralized, i.e., must be a linear combination of good (i.e., not of the bad type) brackets of lower l-degree at the equilibrium. 
Consider system (3) and (4) and rewrite the drift and control vector fields as

$f=\left(\dot{q}_{1}, \dot{q}_{2}, 0, R(q, \dot{q})\right), \quad g_{j}=\left(0,0, e_{j}, J_{j}(q)\right), \quad j \in I_{m}$

where $I_{m}$ denotes the set $\{1, \cdots, m\}$.

The following Lie bracket calculations are straightforward:

$$
\begin{array}{rlrl}
{\left[g_{j}, g_{i}\right]} & \equiv 0, i, j \in I_{m} & \\
{\left[f, g_{i}\right]} & =\left(-e_{i},-J_{i}(q), 0, *\right), \quad & i \in I_{m} \\
{\left[g_{j},\left[f, g_{i}\right]\right]} & =\left(0,0,0, H_{i j}(q)\right), \quad i, j \in I_{m} \\
{\left[f,\left[g_{j},\left[f, g_{i}\right]\right]\right]} & =\left(0,-H_{i j}(q), 0, *\right), \quad i, j \in I_{m}
\end{array}
$$

where

$$
\begin{aligned}
H_{i j}(q)= & \frac{\partial J_{i}(q)}{\partial q} h_{j}(q)+\frac{\partial J_{j}(q)}{\partial q} h_{i}(q) \\
& -\frac{\partial}{\partial \dot{q}}\left(\frac{\partial R(q, \dot{q})}{\partial \dot{q}} h_{i}(q)\right) h_{j}(q), \quad i, j \in I_{m} \\
h_{i}(q)= & \left(\begin{array}{c}
e_{i} \\
J_{i}(q)
\end{array}\right), \quad i \in I_{m} .
\end{aligned}
$$

Note that the vertical lift of $h_{i}$ (considered as a vector field on the configuration space $\mathbf{Q}$ ) is the control vector field $g_{i}$. Note also that $H_{i j}(q)=H_{j i}(q), \forall q \in \mathbf{Q}, \forall i, j \in I_{m}$.

We now present the following result which is a generalization of the results in [18] and [25].

Theorem 3: Let $n-m \geq 1$ and let $\left(q^{e}, 0\right)$ denote an equilibrium solution. The underactuated mechanical system, defined by (3) and (4), is small time locally controllable at $\left(q^{e}, 0\right)$ if there exists a set of $n-m$ pairs of indexes $\left(i_{k}, j_{k}\right) \in I_{m}^{2}, i_{k} \neq j_{k}, k \in I_{n-m}$, such that

$$
\operatorname{dimspan}\left\{H_{i_{k} j_{k}}\left(q^{e}\right), k \in I_{n-m}\right\}=n-m
$$

and

$$
H_{i_{k} i_{k}}\left(q^{e}\right)=0, \quad \forall k \in I_{n-m} .
$$

Proof: Consider system (3) and (4) and assume that (18) and (19) hold. By (18), the space spanned by the vectors

$$
\begin{gathered}
g_{i},\left[f, g_{i}\right],\left[g_{j_{k}},\left[f, g_{i_{k}}\right]\right],\left[f,\left[g_{j_{k}},\left[f, g_{i_{k}}\right]\right]\right], \\
\quad i \in I_{m}, k \in I_{n-m}
\end{gathered}
$$

has dimension $2 n$ at $\left(q^{e}, 0\right)$, and hence the system is strongly accessible at $\left(q^{e}, 0\right)$. Let $l_{0}=l_{i_{k}}=2, k \in I_{n-m}$, and $l_{i}=3, i \neq i_{k}, i \in I_{m}, k \in I_{n-m}$. The value $\delta(B)=$ $\sum_{i=0}^{m} \delta^{i}(B)$ of a bad bracket must necessarily be odd. Any bad bracket with $\delta(B) \geq 5$ has 1 -degree greater than or equal to ten. Clearly, these brackets are l-neutralized since the spanning good brackets (20) have l-degree less than ten. Hence, it suffices to show that bad brackets with $\delta(B)=1$ and $\delta(B)=3$ are l-neutralized. The only bad bracket with $\delta(B)=1$ is $f$, which vanishes at the equilibrium. By (19), the bad brackets $\left[g_{i_{k}},\left[f, g_{i_{k}}\right]\right], k \in I_{n-m}$ are all zero at $\left(q^{e}, 0\right)$; and by (18), the bad brackets $\left[g_{i},\left[f, g_{i}\right]\right], i \neq i_{k}, i \in I_{m}, k \in$ $I_{n-m}$, can be written as linear combinations of the good brackets $\left[g_{j_{k}},\left[f, g_{i_{k}}\right]\right], k \in I_{n-m}$, which have lesser l-degree. It follows that the Bianchini and Stefani condition is satisfied at $\left(q^{e}, 0\right)$. Hence, under the stated assumptions, system (3) and (4) is small time locally controllable at $\left(q^{e}, 0\right)$.

Reference [18, Proposition 3] contains the result in Theorem 3 for the special case of one unactuated degree of freedom only, i.e., $n-m=1$. Theorem 3 is also more general than our previous result in [25] that does apply for the case $n-m \geq 1$.

Note that for (18) to hold the condition $m(m+1) \geq 2 n$ must be satisfied. This condition arises due to the fact that in the above result we have considered Lie brackets up to degree four only. It is possible to develop a result which weakens or even removes this restriction by also taking into account higher order Lie brackets. Such a development is relatively straightforward for underactuated mechanical systems with no potential or frictional forces, i.e., systems for which the components of $R(q, \dot{q})$ are of second-order in the $\dot{q}$-variables.

We now restrict our consideration to underactuated mechanical systems with no potential or friction forces. As shown in Lewis and Murray [17], for such systems, when evaluated at the equilibrium the only nontrivial brackets are those satisfying $\sum_{i=1}^{m} \delta^{i}(B)-\delta^{0}(B)=0$ or $\sum_{i=1}^{m} \delta^{i}(B)-\delta^{0}(B)=1$. Clearly, the brackets with $\sum_{i=1}^{m} \delta^{i}(B)-\delta^{0}(B)=0$ are all good, and the only bad brackets are those with $\sum_{i=1}^{m} \delta^{i}(B)-$ $\delta^{0}(B)=1, \delta^{0}(B)$ odd and $\delta^{i}(B)$ even, $\forall i \in I_{m}$.

Borrowing ideas from Lewis and Murray [17], we define the following sequence of collections of vector fields:

$$
\begin{aligned}
\mathcal{G}_{1} & =\left\{g_{i}, i \in I_{m}\right\} \\
\mathcal{G}_{k} & =\left\{[X,[f, Y]], X \in \mathcal{G}_{i}, Y \in \mathcal{G}_{j}, k=i+j\right\}, \quad k \geq 2 \\
\mathcal{G} & =\bigcup_{i \geq 2} \mathcal{G}_{i} .
\end{aligned}
$$

Let $X$ denote a vector field in $\mathcal{G}$. It is easy to show that $X$ has the form $X=(0,0,0, A(q))$, where $A(q)$ is an $n-m$ vector function and its Lie bracket with $f$ can be written as $[f, X]=(0,-A(q), 0, *)$. Now let $\left(q^{e}, 0\right)$ denote an equilibrium solution. Clearly, if there exists an integer $k^{*} \geq 2$ such that

$$
\operatorname{dim} \operatorname{span}\left\{X\left(q^{e}, 0\right), X \in \bigcup_{i=2}^{k^{*}} \mathcal{G}_{i}\right\}=n-m
$$

then the system is strongly accessible at $\left(q^{e}, 0\right)$, i.e., the system satisfies a necessary condition for STLC at $\left(q^{e}, 0\right)$. As shown in [17], all the bad brackets can be written as linear combinations of the bad brackets contained in $\mathcal{G}$. Thus, a sufficient condition for STLC at $\left(q^{e}, 0\right)$ can be obtained by considering the bad brackets in $\mathcal{G}$ and applying the Bianchini and Stefani condition [3].

The following result can now be stated.

Theorem 4: Let $n-m \geq 1$ and let $\left(q^{e}, 0\right)$ denote an equilibrium solution. Consider the underactuated mechanical system, defined by (3) and (4), and assume that the components of $R(q, \dot{q})$ are of second-order in $\dot{q}$-variables. Also assume that (21) is satisfied. Then, the system is small time locally controllable at $\left(q^{e}, 0\right)$ if there exists an admissible weight vector $\mathbf{l}=\left(l_{0}, l_{1}, \cdots, l_{m}\right), l_{i} \geq l_{0} \geq 0, \forall i$, such that every bad bracket in $\mathcal{G}_{2 k}, k \in \mathbf{Z}^{+}$, can be l-neutralized.

In the next section, selected examples of underactuated mechanical systems are studied to illustrate the above theoretical 


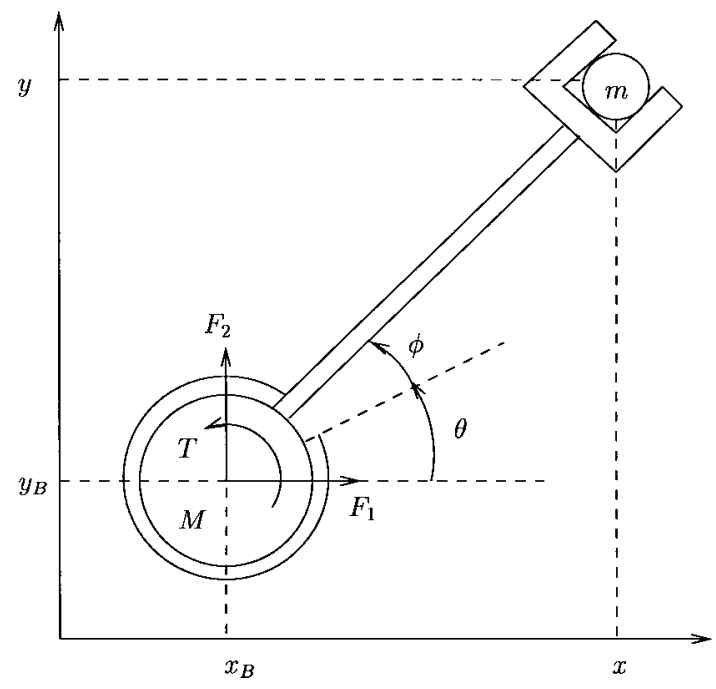

Fig. 1. Model of an underactuated manipulator.

results. The examples are described in a physical context, and the theoretical results of this paper are used to expose the fundamental control properties of these examples. Each example is nontrivial. The first example is a new control theoretical formulation that incorporates a specific design constraint that an elastic mode not be excited. Our second example is an original formulation of a mechanical system with two unactuated degrees of freedom.

\section{Underactuated MeChanical System EXAMPLES}

\section{A. Control of a Manipulator Without Excitation of its Elastic Mode}

Consider a planar PPR robot, i.e., a robot with two prismatic and one revolute joint, moving on a horizontal plane so that gravity can be ignored. Assume that the two prismatic joints are rigid, whereas the revolute joint is coupled to the endeffector through an elastic degree of freedom. Also assume that all the joints are actuated. An idealized model of this manipulator [2] is shown in Fig. 1. The model consists of a base body, which can translate and rotate freely in the plane, and a massless arm at the tip of which the end-effector is attached. The base body is connected to the massless arm by a linear torsional spring whose neutral position is $\phi=0$. The Cartesian position $\left(x_{b}, y_{b}\right)$ of the base body as well as the angle $\theta$ through which the base body is rotated can be controlled. The variable $\phi$ measures the deviation of the massless arm from the assigned value $\theta$. Whenever the variable $\phi$ is displaced from zero, it induces a restoring torque $-k \phi$, where $k$ denotes the torsional spring constant. Let $(x, y)$ denote the end-effector position of the manipulator. Also, let the base body have mass $M$ and rotational inertia $I$, the endeffector and payload combination have mass $m$, and let $l$ be the length of the massless arm.

Assume that initially $\phi(0)=\dot{\phi}(0)=0$. The control problem is to move the manipulator between any given initial configuration $\left(x^{0}, y^{0}, \theta^{0}\right)$ and final configuration $\left(x^{f}, y^{f}, \theta^{f}\right)$ such that no elastic deformation occurs, i.e., $\phi(t)=0, \forall t \geq 0$.
We use the ideas introduced previously to formulate the above problem as a nonlinear control problem. Let $\left(F_{1}, F_{2}, T\right) \in \mathbf{R}^{3}$ denote the vector of control inputs applied to the base body, where $\left(F_{1}, F_{2}\right)$ are the force inputs in the $x$ and $y$ direction, respectively, and $T$ is the torque input. Then we have a Lagrangian system with the Lagrangian

$$
\begin{aligned}
L= & \frac{1}{2}(M+m)\left(\dot{x}^{2}+\dot{y}^{2}\right)+\frac{1}{2} I \dot{\theta}^{2}+\frac{1}{2} M l^{2}(\dot{\theta}+\dot{\phi})^{2}-\frac{1}{2} k \phi^{2} \\
& +M l(\dot{\theta}+\dot{\phi})(\dot{x} s(\theta+\phi)-\dot{y} c(\theta+\phi))
\end{aligned}
$$

where $c(\cdot)=\cos (\cdot)$ and $s(\cdot)=\sin (\cdot)$. The virtual work is given by

$$
\delta W=F_{1} \delta(x-l c(\theta+\phi))+F_{2} \delta(y-l s(\theta+\phi))+T \delta \theta .
$$

Equations of motion can then be written as

$$
\begin{aligned}
& (M+m) \ddot{x}+M l(\ddot{\theta}+\ddot{\phi}) s(\theta+\phi)+M l(\dot{\theta}+\dot{\phi})^{2} c(\theta+\phi) \\
& \quad=F_{1} \\
& (M+m) \ddot{y}-M l(\ddot{\theta}+\ddot{\phi}) c(\theta+\phi)+M l(\dot{\theta}+\dot{\phi})^{2} s(\theta+\phi) \\
& \quad=F_{2} \\
& M l(\ddot{x} s(\theta+\phi)-\ddot{y} c(\theta+\phi))+\left(I+M l^{2}\right) \ddot{\theta}+M l^{2} \ddot{\phi} \\
& \quad=l\left(F_{1} s(\theta+\phi)-F_{2} c(\theta+\phi)\right)+T \\
& M l(\ddot{x} s(\theta+\phi)-\ddot{y} c(\theta+\phi))+M l^{2} \ddot{\theta}+M l^{2} \ddot{\phi}+k \phi \\
& \quad=l\left(F_{1} s(\theta+\phi)-F_{2} c(\theta+\phi)\right) .
\end{aligned}
$$

Setting first $\phi \equiv 0$ and then rearranging the resulting equations, we obtain

$$
\begin{aligned}
(M+m) \ddot{x}+M l \ddot{\theta} \sin \theta+M l \dot{\theta}^{2} \cos \theta & =F_{1} \\
(M+m) \ddot{y}-M l \ddot{\theta} \cos \theta+M l \dot{\theta}^{2} \sin \theta & =F_{2} \\
I \ddot{\theta} & =T \\
\ddot{x} \sin \theta-\ddot{y} \cos \theta & =0 .
\end{aligned}
$$

It is easy to check that (29) satisfies Definition 1 and hence represents a nonintegrable acceleration relation, which implies that the end-effector acceleration exerts no torque on the elastic joint. This condition can be viewed as a design constraint.

In order to satisfy the above equations, it is required to select

$$
T=\frac{I}{M l}\left(F_{1} \sin \theta-F_{2} \cos \theta\right) .
$$

It is then straightforward to show that the above equations can be equivalently written as

$$
\begin{aligned}
& \ddot{x}=u_{1} \\
& \ddot{\theta}=u_{2} \\
& \ddot{y}=u_{1} \tan \theta
\end{aligned}
$$

where

$$
\begin{aligned}
u_{1}= & \frac{1}{M+m}\left(F_{1} \cos \theta+F_{2} \sin \theta\right) \cos \theta \\
& -\frac{M l}{M+m} \dot{\theta}^{2} \cos \theta \\
u_{2}= & \frac{1}{M l}\left(F_{1} \sin \theta-F_{2} \cos \theta\right) .
\end{aligned}
$$

Note that now the control problem is reduced to designing controls $u_{1}$ and $u_{2}$ for system (31)-(33). Once these controls 
are designed, one can use relations (34) and (35) to determine the controls $F_{1}$ and $F_{2}$. Finally, $T$ can be determined from (30).

Let $(q, \dot{q})=(x, \theta, y, \dot{x}, \dot{\theta}, \dot{y}) \in \mathbf{M}=\mathbf{R} \times(-\pi / 2, \pi / 2) \times$ $\mathbf{R} \times \mathbf{R}^{3}$ denote the state. Then, the drift and control vector fields on $\mathbf{M}$ are given by

$$
\begin{aligned}
f & =\dot{x} \frac{\partial}{\partial x}+\dot{\theta} \frac{\partial}{\partial \theta}+\dot{y} \frac{\partial}{\partial y} \\
g_{1} & =\frac{\partial}{\partial \dot{x}}+\tan \theta \frac{\partial}{\partial \dot{y}}, \quad g_{2}=\frac{\partial}{\partial \dot{\theta}} .
\end{aligned}
$$

Clearly, the set of equilibrium solutions corresponding to $u=0$ is given by

$$
\mathbf{M}_{e}=\{(q, \dot{q}) \in \mathbf{M} \mid \dot{q}=0\} .
$$

Note that for this example $n=3$ and $m=2$, and thus there is only one unactuated degree of freedom, i.e., $n-m=1$. The functions $H_{i j}(q), i, j=1,2$, can be determined using (16) as

$$
H_{11}(q) \equiv 0, H_{22}(q) \equiv 0, H_{12}(q)=H_{21}(q)=1+\tan ^{2} \theta .
$$

Clearly, (18) and (19) of Theorem 3 are satisfied at any $\left(q^{e}, 0\right) \in M_{e}$ with $\left(i_{1}, j_{1}\right)=(1,2)$ or $\left(i_{1}, j_{1}\right)=(2,1)$.

We now state the following results which characterize the controllability and stabilizability properties of the constrained manipulator dynamics.

Proposition 1: Let $\mathbf{M}_{e}$ denote the equilibrium manifold and let $\left(q^{e}, 0\right) \in \mathbf{M}_{e}$ denote an equilibrium solution. The following hold for the constrained manipulator dynamics described by (31)-(33).

1) The system is strongly accessible since the space spanned by the vectors

$$
g_{1}, g_{2},\left[f, g_{1}\right],\left[f, g_{2}\right],\left[g_{2},\left[f, g_{1}\right]\right],\left[f,\left[g_{2},\left[f, g_{1}\right]\right]\right]
$$

has dimension six at any $(q, \dot{q}) \in \mathbf{M}$.

2) The system is small time locally controllable at $\left(q^{e}, 0\right)$ since the sufficient conditions for STLC of Theorem 3 are satisfied.

3) There exist both time-invariant piecewise analytic feedback laws and time-periodic continuous feedback laws which asymptotically stabilize $\left(q^{e}, 0\right)$.

4) There is no time-invariant continuous feedback law which asymptotically stabilizes the closed loop to $\left(q^{e}, 0\right)$.

Obviously, the controllability properties given in Proposition 1 guarantee the existence of the solution to the problem of controlling the manipulator with zero elastic deformation. Time-invariant discontinuous feedback control laws have been developed for this problem in [28] and [29] based on the above theoretical results.

Note that the results given in Proposition 1 are valid only for the constrained manipulator dynamics described by (31)-(33); they do not imply that the original mechanical system (22)-(25) is STLC at the equilibrium $(q, \phi, \dot{q}, \dot{\phi})=$ $\left(q^{e}, \phi^{e}, 0,0\right)$ nor that the equilibrium can be asymptotically stabilized by means of a feedback law derived on the basis of system (31)-(33). Note also that the restoring torque $-k \phi$ plays no role in the derivation or validity of these results, i.e.,

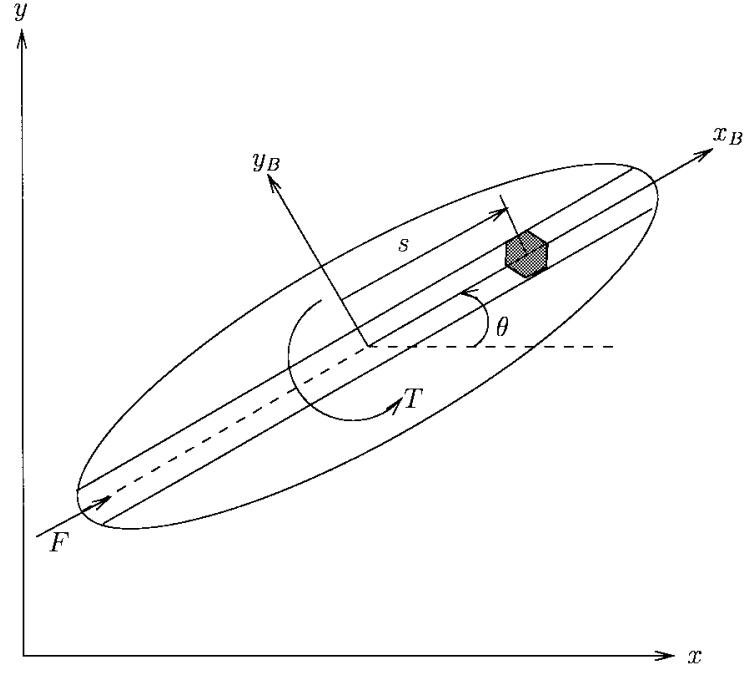

Fig. 2. Model of a rigid body containing a sliding block.

the above results remain true even if the torsional spring is removed.

\section{B. Control of a Planar Rigid Body Containing a Sliding Block}

Consider the planar rigid body shown in Fig. 2, moving in a horizontal plane so that gravity can be ignored. The rigid body can translate and rotate freely in the plane. Let $x_{B} y_{B}$ be a body-fixed orthonormal frame (B-frame) whose origin is located at the center of mass of the body. Assume that a block moves along the $x_{B}$-axis in a smooth slot in the rigid body. Let $(x, y)$ denote the position of the center of mass of the rigid body and let $\theta$ be the orientation of the rigid body. Also, let $s \in(-l / 2, l / 2)$, where $l$ is a positive constant, denote the position of the block relative to the center of mass of the rigid body. Assume that the rigid body has mass $M$ and rotational inertia $I$, the block has mass $m$ and rotational inertia $I_{b}$.

Let $F$ and $T$ denote the external force (along the $x_{B}$-axis) and the external torque (about the center of mass of the body), respectively, and let $(\xi, \eta)$ denote the position of the center of mass of the body in the B-frame, which is given by

$$
\begin{aligned}
& \xi=x \cos \theta+y \sin \theta \\
& \eta=-x \sin \theta+y \cos \theta .
\end{aligned}
$$

Then, the equations of motion can be written in $(\theta, \xi, \eta, s)$ coordinates as

$$
\begin{aligned}
& {\left[I+I_{b}+M\left(\xi^{2}+\eta^{2}\right)+m\left((\xi+s)^{2}+\eta^{2}\right)\right] \ddot{\theta}-(M+m) \eta \ddot{\xi}} \\
& \quad+[(M+m) \dot{\xi}+m s] \ddot{\eta}-m \eta \ddot{s}+2(M+m) \\
& \quad \cdot(\xi \dot{\xi}+\eta \dot{\eta}) \dot{\theta}+2 m(\xi \dot{\xi}+s \dot{\xi}+s \dot{s}) \dot{\theta}=T \\
& -(M+m) \eta \ddot{\theta}+(M+m) \ddot{\xi}+m \ddot{s}-2(M+m) \dot{\eta} \dot{\theta} \\
& \quad-(M+m) \dot{\xi} \dot{\theta}^{2}-m s \dot{\theta}^{2}=F \\
& \quad[(M+m) \xi+m s] \ddot{\theta}+(M+m) \ddot{\eta}+2(M+m) \dot{\xi} \dot{\theta} \\
& \quad+2 m \dot{s} \dot{\theta}-(M+m) \eta \dot{\theta}^{2}=0 \\
& \ddot{\xi}+\ddot{s}-\eta \ddot{\theta}-2 \dot{\eta} \dot{\theta}-\xi \dot{\theta}^{2}-s \dot{\theta}^{2}=0 .
\end{aligned}
$$

It is easy to check that (38) and (39) satisfy Definition 1 and hence represent two nonintegrable acceleration relations. 
Clearly, the above equations can be written as

$$
\begin{aligned}
& \ddot{\theta}=u_{1} \\
& \ddot{\xi}=u_{2} \\
& \ddot{\eta}=-(\dot{\xi}+a s) u_{1}-2 \dot{\xi} \dot{\theta}-2 a \dot{s} \dot{\theta}+\eta \dot{\theta}^{2} \\
& \ddot{s}=\eta u_{1}-u_{2}+2 \dot{\eta} \dot{\theta}+\xi \dot{\theta}^{2}+s \dot{\theta}^{2}
\end{aligned}
$$

where $a=m /(M+m)$ and

$$
\begin{aligned}
u_{1}= & \frac{1}{I+I_{b}+a M s^{2}}(T+\eta F-2 a M \eta s \dot{s} \dot{\theta}) \\
u_{2}= & \frac{1}{I+I_{b}+a M s^{2}} \\
& \cdot\left[\eta T+\left(\frac{I+I_{b}}{M}+a s^{2}+\eta^{2}\right) F-2 a M s \dot{s} \dot{\theta}\right] \\
& +2 \dot{\eta} \dot{\theta}+\xi \dot{\theta}^{2} .
\end{aligned}
$$

Let $(q, \dot{q})=(\theta, \xi, \eta, s, \dot{\theta}, \dot{\xi}, \dot{\eta}, \dot{s}) \in \mathbf{M}=\mathbf{S}^{1} \times \mathbf{R}^{2} \times$ $(-l / 2, l / 2) \times \mathbf{R}^{4}$ denote the state. Then, the drift and control vector fields on $\mathbf{M}$ are given by

$$
\begin{aligned}
f= & \dot{\theta} \frac{\partial}{\partial \theta}+\dot{\xi} \frac{\partial}{\partial \dot{\xi}}+\dot{\eta} \frac{\partial}{\partial \eta}+\dot{s} \frac{\partial}{\partial s}+\left(\eta \dot{\theta}^{2}-2 \dot{\xi} \dot{\theta}-2 a \dot{s} \dot{\theta}\right) \frac{\partial}{\partial \dot{\eta}} \\
& +\left(2 \dot{\eta} \dot{\theta}+\xi \dot{\theta}^{2}+s \dot{\theta}^{2}\right) \frac{\partial}{\partial \dot{s}} \\
g_{1}= & \frac{\partial}{\partial \dot{\theta}}-(\xi+a s) \frac{\partial}{\partial \dot{\eta}}+\eta \frac{\partial}{\partial \dot{s}}, \quad g_{2}=\frac{\partial}{\partial \dot{\xi}}-\frac{\partial}{\partial \dot{s}}
\end{aligned}
$$

Clearly, the set of equilibrium solutions corresponding to $u=0$ is given by

$$
\mathbf{M}_{e}=\{(q, \dot{q}) \in \mathbf{M} \mid \dot{q}=0\}
$$

Note that for this example $n=4$ and $m=2$, and thus there are two unactuated degrees of freedom, i.e., $n-m=2$. The following Lie bracket calculations are straightforward:

$$
\begin{aligned}
{\left[g_{1},\left[f, g_{1}\right]\right] } & =2(a-1) \eta \frac{\partial}{\partial \dot{\eta}}+2(a-1) s \frac{\partial}{\partial \dot{s}} \\
{\left[g_{2},\left[f, g_{2}\right]\right] } & \equiv 0 \\
{\left[g_{2},\left[f, g_{1}\right]\right] } & =(1-a) \frac{\partial}{\partial \dot{\eta}} \\
{\left[g_{1},\left[f,\left[g_{2},\left[f, g_{1}\right]\right]\right]\right] } & =(a-1) \frac{\partial}{\partial \dot{s}} .
\end{aligned}
$$

Clearly, the condition (21) of Theorem 4 is satisfied at any $\left(q^{e}, 0\right) \in M_{e}$ with $k^{*}=3$. Let $\mathbf{l}=\left(l_{0}, l_{1}, l_{2}\right)=(2,3,2)$ be the weight vector. It is easy to show that any bad bracket in $\mathcal{G}_{2 k}, k \in \mathbf{Z}^{+}$is a linear combination of good brackets of lower l-degree at any equilibrium $\left(q^{e}, 0\right) \in M_{e}$ with $s^{e}=0$.

The following results characterize the controllability and stabilizability properties of the dynamics of the rigid body and the sliding block.

Proposition 2: Let $\mathbf{M}_{e}$ denote the equilibrium manifold and let $\left(q^{e}, 0\right) \in \mathbf{M}_{e}$ denote an equilibrium solution with $s^{e}=0$. The following hold for the dynamics of the rigid body and the sliding block, described by (40)-(43).

1) The system is strongly accessible since the space spanned by the vectors

$g_{1}, g_{2},\left[f, g_{1}\right],\left[f, g_{2}\right],\left[g_{2},\left[f, g_{1}\right]\right],\left[f,\left[g_{2},\left[f, g_{1}\right]\right]\right]$ $\left[g_{1},\left[f,\left[g_{2},\left[f, g_{1}\right]\right]\right]\right],\left[f,\left[g_{1},\left[f,\left[g_{2},\left[f, g_{1}\right]\right]\right]\right]\right]$

has dimension eight at any $(q, \dot{q}) \in \mathbf{M}$.

2) The system is small time locally controllable at $\left(q^{e}, 0\right)$ since the sufficient conditions for STLC of Theorem 4 are satisfied.

3) There exist both time-invariant piecewise analytic feedback laws and time-periodic continuous feedback laws which asymptotically stabilize $\left(q^{e}, 0\right)$.

4) There is no time-invariant continuous feedback law which asymptotically stabilizes the closed loop to $\left(q^{e}, 0\right)$.

Obviously, the controllability properties given in Proposition 2 guarantee the existence of the solution to the problem of controlling the rigid body and the sliding block. Clearly, if the block is coupled to the rigid body through an elastic degree of freedom, the above control problem is equivalent to controlling both the three rigid body modes and the elastic mode.

\section{CONCLUSIONS}

A theoretical framework has been presented for the dynamics and control of underactuated mechanical systems which satisfy nonintegrable acceleration relations. In particular, a nonlinear control system formulation has been introduced and certain controllability and stabilizability properties have been analyzed. These fundamental properties should provide a foundation for further research in this area.

We believe that motion planning algorithms and feedback stabilization schemes can be developed for the class of underactuated systems with nonintegrable acceleration relations, just as such developments have been made for classical nonholonomic control systems (mechanical systems with nonintegrable velocity relations) [15]. For example, specific feedback stabilization schemes have recently been developed for the control of an underactuated surface vessel [24], [27] for hover control of an V/STOL aircraft [19], for the control of the RTAC system [16], and for the control of a rigid body with an unactuated internal degree of freedom [20], [30]. These papers use time-invariant discontinuous feedback laws developed based on a nonsmooth state transformation, time-invariant discontinuous feedback laws developed based on introduction of a piecewise constant switching signal, and nonsmooth time periodic feedback laws. These particular feedback stabilization approaches, and other approaches that have been introduced for classical nonholonomic control systems, can perhaps be extended to the class of underactuated mechanical systems with nonintegrable acceleration relations. These extensions are not direct, but the results in [9], [16], [18]-[21], and [27]-[29] are encouraging. 


\section{ACKNOWLEDGMENT}

The authors wish to acknowledge the support provided by the University of Twente, which enabled the visits to the University by the first author during the academic year $1995-1996$ and by the third author during February, 1996. The authors also wish to acknowledge suggestions of the reviewers that helped to refine the statement of Theorem 3.

\section{REFERENCES}

[1] H. Arai and S. Tachi, "Position control of a manipulator with passive joints using dynamic coupling," IEEE Trans. Robot. Automat., vol. 7, pp. 528-534, 1991

[2] J. Baillieul, "Kinematically redundant robots with flexible components," IEEE Contr. Syst. Mag., vol. 13, pp. 15-21, 1993.

[3] R. M. Bianchini and G. Stefani, "Controllability along a trajectory: A variational approach," SIAM J. Contr. Optimiz., vol. 31, no. 4, pp. 900-927, 1993.

[4] A. M. Bloch, M. Reyhanoglu, and N. H. McClamroch, "Control and stabilization of nonholonomic dynamic systems," IEEE Trans. Automat. Contr., vol. 37, pp. 1746-1757, Nov. 1992.

[5] R. W. Brockett, "Asymptotic stability and feedback stabilization," Differential Geometric Control Theory, R. W. Brockett, R. S. Millman, and H. J. Sussmann, Eds. Boston, MA: Birkhauser, 1983, pp. 181-191.

[6] R. T. Bupp, C.-J. Wan, V. T. Coppola, and D. S. Bernstein, "Design of a rotational actuator for global stabilization of a translational motion," in Proc. Symp. Active Control of Vibration and Noise, ASME Winter Meeting, 1994.

[7] F. H. Clarke, Yu. S. Ledyaev, E. D. Sontag, and A. I. Subbotin, "Asymptotic controllability implies feedback stabilization," IEEE Trans. Automat. Contr., vol. 42, pp. 1394-1407, Oct. 1997.

[8] J.-M. Coron, "On the stabilization in finite time of locally controllable systems by means of continuous time-varying feedback law," SIAM J. Contr. Optimiz., vol. 33, no. 3, pp. 804-833, 1995.

[9] J.-M. Coron and E.-Y. Kerai, "Explicit feedbacks stabilizing the attitude of a rigid spacecraft with two control torques," Automatica, vol. 32, no. 5, pp. 669-677, 1996.

[10] O. Egeland, M. Dalsmo, and O. J. Sordalen, "Feedback control of a nonholonomic underwater vehicle with constant desired configuration," Int. J. Res., vol. 15, pp. 24-35, 1996.

[11] D. T. Greenwood, Principles of Dynamics. Englewood Cliffs, NJ: Prentice-Hall, 1988.

[12] Y.-L. Gu, "A direct adaptive control scheme for underactuated dynamic systems," in Proc. IEEE Conf. Decision and Control, San Antonio, TX, 1993, pp. 1625-1627.

[13] J. Hauser, S. Sastry, and G. Meyer, "Nonlinear control design for slightly nonminimum phase systems: Application to V/STOL aircraft," Automatica, vol. 28, no. 4, pp. 665-679, 1992.

[14] M. Jankovic, D. Fontaine, and P. V. Kokotovic, "TORA example: Cascade and passivity-based control designs," IEEE Trans. Contr. Syst. Tech., vol. 4, no. 3, pp. 292-297, 1996.

[15] I. Kolmanovsky and N. H. McClamroch, "Developments in nonholonomic control problems," IEEE Contr. Syst. Mag., vol. 15, no. 6, pp. 20-36, 1995.

[16] _ "Hybrid feedback stabilization of rotational translational actuator (RTAC) system," Int. J. Robust and Nonlinear Contr., vol. 8, pp. 367-375, 1998.

[17] A. D. Lewis and R. M. Murray, "Configuration controllability of simple mechanical control systems," SIAM J. Contr. Optimiz., vol. 35, no. 3, pp. 766-790, 1997.

[18] A. De Luca, R. Mattone, and G. Oriolo, "Steering a class of redundant mechanisms through end-effector generalized forces," IEEE Trans. Robot. Automat., vol. 14, no. 2, pp. 329-333, 1998.

[19] N. H. McClamroch and I. Kolmanovsky, "A hybrid switched mode control for V/STOL flight control problems," in Proc. IEEE Conf. Decision and Control, Kobe, Japan, 1996, pp. 2648-2653.

[20] N. H. McClamroch, C. Rui, I. Kolmanovsky, S. Cho, and M. Reyhanoglu, "Planar maneuvers of a rigid body with an unactuated internal degree of freedom," in Proc. Amer. Control Conf., Philadelphia, PA, 1998, pp. 229-233.
[21] P. Morin and C. Samson, "Time-varying exponential stabilization of the attitude of a rigid spacecraft with two controls," IEEE Trans. Automat. Contr., vol. 42, pp. 528-533, Apr. 1997.

[22] H. Nijmeijer and A. J. van der Schaft, Nonlinear Dynamical Control Systems. New York: Springer-Verlag, 1990.

[23] G. Oriolo and Y. Nakamura, "Control of mechanical systems with second-order nonholonomic constraints: Underactuated manipulators," in Proc. IEEE Conf. Decision and Control, Brighton, U.K., 1991, pp. 2398-2403.

[24] K. Y. Pettersen and O. Egeland, "Exponential stabilization of an underactuated surface vessel," in Proc. IEEE Conf. Decision and Control, Kobe, Japan, 1996, pp. 967-971.

[25] M. Reyhanoglu and A. J. van der Schaft, "Dynamics and control of second-order nonholonomic systems," in Benelux Meeting, Mierlo, The Netherlands, Mar. 1996

[26] M. Reyhanoglu, A. J. van der Schaft, N. H. McClamroch, and I. Kolmanovsky, "Nonlinear control of a class of underactuated systems," in Proc. IEEE Conf. Decision and Control, Kobe, Japan, 1996, pp. $1682-1687$.

[27] M. Reyhanoglu, "Exponential stabilization of an underactuated autonomous surface vessel," Automatica, vol. 33, no. 12, pp. 2249-2254, 1997.

[28] _ "Control of a super-articulated robot manipulator with joint elasticity," in Proc. Int. Conf. Control and Automat. Robotics Vision, Westin Stamford, Singapore, 1996, pp. 172-176.

[29] __ "Feedback control of a flexible joint robot," in Proc. European Control Conf., Brussels, Belgium, July 1997.

[30] M. Reyhanoglu, S. Cho, N. H. McClamroch, and I. Kolmanovsky, "Discontinuous feedback control of a planar rigid body with an unactuated internal degree of freedom," in Proc. IEEE Conf. Decision and Control, Tampa, FL, 1998, pp. 433-438.

[31] E. P. Ryan, "On Brockett's condition for smooth stabilizability and its necessity in a context of nonsmooth feedback," SIAM J. Contr. Optimiz., vol. 32, no. 6, pp. 1597-1604, 1994.

[32] M. W. Spong, "The swing up control problem for the acrobot," IEEE Contr. Syst. Mag., vol. 15, pp. 49-55, 1995.

[33] _ "Energy based control of a class of underactuated mechanical systems," in Proc. IFAC World Congr., San Francisco, CA, 1996, pp. 431-435.

[34] C.-Yi. Su and Y. Stepanenko, "Sliding mode control of nonholonomic mechanical systems: Underactuated manipulators case," in Proc. IFAC Nonlinear Contr. Syst. Design, 1995, Tahoe City, CA, pp. 609-613.

[35] H. J. Sussmann, "A general theorem on local controllability," SIAM J. Contr. Optimiz., vol. 25, no. 1, pp. 158-194, 1987.

[36] __ "Subanalytic sets and feedback control," J. Diff. Equations, vol. 31, pp. 31-52, 1979.

[37] K. Y. Wichlund, O. J. Sordalen, and O. Egeland, "Control of vehicles with second-order nonholonomic constraints: Underactuated vehicles," in Proc. European Control Conf., Rome, Italy, 1995, pp. 3086-3091.

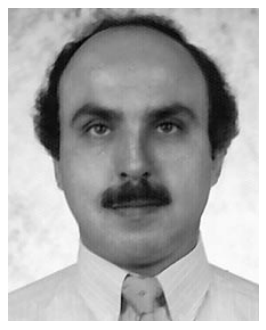

Mahmut Reyhanoglu (S'91-M'92) received the B.S. degree in aeronautical engineering and the M.S. degree in mechanical engineering from Istanbul Technical University, Turkey, in 1982 and 1984, respectively. He also received the M.S. degree in aeronautical and astronautical engineering from the Ohio State University, Columbus, the M.S. degree in electrical engineering systems, and the Ph.D. degree in aerospace engineering from the University of Michigan, Ann Arbor, in 1987, 1991, and 1992, respectively.

From 1992 to 1998, he held visiting appointments in the Department of Mechanical Engineering at the King Fahd University of Petroleum and Minerals, Dhahran, Saudi Arabia; in the Department of Applied Mathematics at the University of Twente, Enschede, Netherlands; and in the Department of Mechanical Engineering at Ohio University, Athens. He is currently with the Department of Physical Sciences at Embry-Riddle Aeronautical University, Daytona Beach, FL. His major research interests include the areas of nonlinear dynamical systems, controls, and robotics, with particular emphasis on applications to mechanical systems and aerospace systems. 


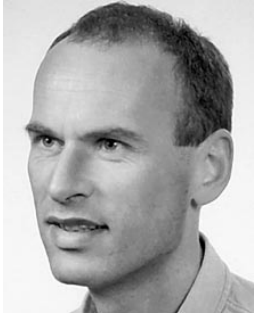

Arjan van der Schaft (M'91-SM'98) was born in Vlaardingen, The Netherlands, in 1955. He received the undergraduate and $\mathrm{Ph} . \mathrm{D}$. degrees in mathematics from the University of Groningen, The Netherlands, in 1979 and 1983, respectively.

In 1982 he joined the Faculty of Mathematical Sciences, University of Twente, Enschede, The Netherlands, where he is presently an Associate Professor. His research interests include the mathematical modeling of physical and engineering systems and the control of nonlinear and hybrid systems.

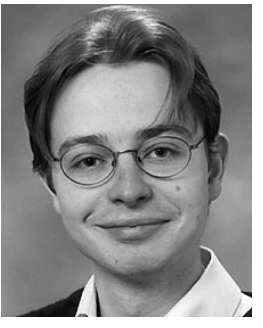

Ilya Kolmanovsky (S'94-M'95) studied as an undergraduate student at the Moscow Aviation Institute, Russia. He received the M.S. and Ph.D. degrees in aerospace engineering and the M.S. degree in mathematics from the University of Michigan, Ann Arbor.

$\mathrm{He}$ is presently working on powertrain modeling and control at the Ford Research Laboratory, Dearborn, MI. On the theoretical side, his interests include the area of nonlinear control.

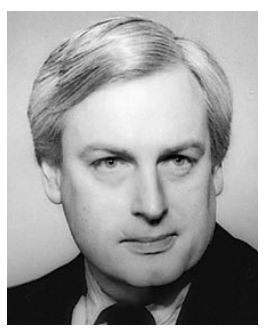

N. Harris McClamroch (S'68-M'68-SM'86F'88) received the Ph.D. degree in engineering mechanics from The University of Texas, Austin.

Since 1967, he has been at The University of Michigan, Ann Arbor, where he is currently a Professor in the Department of Aerospace Engineering. His main research interests include stability and control of nonlinear systems, especially as applied to aerospace vehicles. He is the author of numerous technical papers.

Dr. McClamroch is a past Editor and currently Associate Editor at Large for the IEEE TRAnsactions on Automatic Control; he was President of the Control Systems Society in 1998. 